Background and Objectives B-cell activating factor of the TNF family (BAFF) is important for B cell maturation and plays a role in (auto)antibodies production. Elevated serum levels in relation to autoantibodies were documented in patients with IIM. Promoter region of BAFF gene contains several known sites with single nucleotide polymorphism (SNPs). An association between Rs9514828 (-871 C/T) SNP and susceptibility to idiopathic trombocytopaenic purpura was shown and possible relations to systemic lupus erythematosus, rheumatoid arthritis or Sjögren's syndrome were suggested but in IIM have not been studied yet. Here, we analysed relation of four BAFF SNPs located in the BAFF gene promoter with the development of IIM.

Materials and Methods 146 patients with polymyositis (PM), 150 with dermatomyositis (DM), 11 patients with juvenile DM and 4 patients with inclusion body myositis and 103 healthy individuals were included. Four SNPs located upstream in the BAFF gene (Rs9514827 (-2841 T/C); Rs3759467 (-2704 T/C); Rs1041569 (-2701 T/A); Rs9514828 (-871 C/T)) were analysed by direct DNA sequencing. Serum levels of BAFF (s-BAFF) were evaluated using ELISA. Autoantibodies were detected with immunoprecipitation. The chi square test for analysis of alleles and genotypes associations and SNPStats software for haplotype frequency studies were used. Results Significantly higher frequency of $-2701 \mathrm{~T}$ allele was present in patients $(18 \%)$ compared to healthy controls $(12 \%)(\mathrm{P}=0.029$; OR $1.684(\mathrm{CI} 95 \%=1.050-2.699))$. Additionally, increased $-2841 \mathrm{~T}$ allele $(\mathrm{P}=0.086),-2841 \mathrm{TT}$, CT genotype $(\mathrm{P}=0.066)$ and $-2701 \mathrm{TT}$, AT genotype $(\mathrm{P}=0.079)$ frequencies were observed in patients. SNPs were in strong linkage disequilibrium and formed four common haplotypes (TTAC, CTAT, TCAC, TTTT), with significantly different frequency $(>9 \%)$ distributions between patients and controls (global P-value <0.038). Higher frequency of TTTT haplotype was present in patients $(16.2 \%)$ compared to controls $(9.3 \%$; OR 1.99 (95\% CI 1.15-3.47; $\mathrm{P}<0.015)$ ) relative to the most frequent haplotype TTAC. Significantly higher s-BAFF levels were detected in patients compared to healthy controls $(\mathrm{P}<0.0001)$ and in patients with anti-Jo-1 or anti-PMScl autoantibodies compared to patients without autoantibodies ( $\mathrm{P}=0.028, \mathrm{P}<0.037$ respectively). Higher s-BAFF levels with $-2704 \mathrm{~T}$ allele within anti-Jo-1+ patients $(\mathrm{P}<0.043)$ were found.

Conclusions We describe significant association of SNP (Rs1041569) with myositis and a relation of SNP (Rs3759467) to presence of anti-Jo-1 autoantibodies and s-BAFF levels. These results should be confirmed in an independent cohort of patients and possible relations of s-BAFF levels with disease activity and treatment should be considered.

Acknowledgement IGA -Ministry of Health of the Czech Republic NT/12438-4.

\section{A7.12 IDENTIFICATION OF THE SEMAPHORIN RECEPTOR PLEXIN-A2 AS A CANDIDATE GENE FOR SUSCEPTIBILITY TO ANKYLOSING SPONDYLITIS}

doi:10.1136/annrheumdis-2013-203221.12

A Chatzikyriakidou, PV Voulgari, AA Drosos. Rheumatology Clinic, Department of Internal Medicine, Medical School, University of loannina, Greece

Background and Objectives Semaphorins are a large family of secreted and membrane bound proteins linked to their receptors in a variety of cells and implicated with the development and function of many systems (nevrous, immune, vascular) and organs (bone, heart, lung, kidney). Plexins and neuropilins are the primary semaphorin receptors. The present study addresses Plexin-A2 (PLXNA2) Rs6656560 variant in rheumatoid arthritis (RA), ankylosing spondylitis (AS), and psoriatic arthritis (PsA) susceptibility.

Materials and Methods One hundred thirty-six RA, 49 AS, 29 PsA patients and 147 controls were enrolled in the study. Polymerase chain reaction coupled with restriction fragment length polymorphism analysis was used in Rs6656560 genotyping.

Results Statistical significant difference was observed in Rs6656560 genotypes' distribution between AS patients and controls $(P=0.041)$, but not between RA or PsA patients and controls $(\mathrm{P}=0.183$, $\mathrm{P}=0.729$; respectively). Similarly, significant difference was observed in Rs6656560 alleles' distribution between AS patients and controls $(\mathrm{P}=0.013)$. Since AS and PsA both belong to spondyloarthropathies (SpAs), no statistical difference was observed in genotypes' distribution between these two groups $(\mathrm{P}=0.178)$, while the statistical difference was significant between AS and RA patients $(\mathrm{P}=0.035)$.

Conclusions The positive association of PLXNA2 polymorphism with AS susceptibility seems to indicate its effect in cellular semaphorin signalling related to bone development and remodelling, both of which are implicated in AS features. Additional studies are needed to ensure the revealed genetic association with AS predisposition and the effect of this variant in semaphorin/plexin complex function in specific cell types.

\section{A7.13 IRAK1 RS3027898 POLYMORPHISM: A VARIANT IMPLICATED IN THE PATHOGENESIS OF MORE THAN ONE INFLAMMATORY DISEASE}

doi:10.1136/annrheumdis-2013-203221.13

A Chatzikyriakidou, PV Voulgari, AA Drosos. Rheumatology Clinic, Department of Internal Medicine, Medical School, University of loannina, Greece

Background and Objectives IRAK1 plays significant role in TLR dependent activation of the transcription factor NF-kB, which subsequently increases the expression of many genes such as TNF- $\alpha$ and IL-8 related to immunological reactions. Polymorphism Rs3027898 located in the 3'-untranslated region of IRAK1 gene was studied for its association with rheumatoid arthritis (RA), psoriatic arthritis (PsA) and ankylosing spondylitis (AS) predisposition.

Materials and Methods The polymerase chain reaction-single strand conformation polymorphism analysis coupled with sequencing was used as the screening method for variant genotyping in 136 RA, 29 PsA, and 49 AS patients and 147 controls.

Results IRAK1 polymorphism Rs3027898 was revealed to be associated with all the studied inflammatory conditions. Specifically, strong statistically significant difference was observed in polymorphism distribution between RA patients and controls $(p=0.044)$, PsA patients and controls $(p<0.001)$, and AS patients and controls $(p<0.001)$. Grouping genotypes AA and AC versus $\mathrm{CC}$ the statistical difference was also significant in all groups: RA versus controls $(p=0.017)$, PsA versus controls $(p<0.001)$, and AS versus controls $(p=0.010)$.

Conclusions Here, we report IRAK1 gene polymorphism association with RA, PsA, and AS. This variant, previously, was also related to RA in patients with Chinese origin, with atherothrombotic cerebral infarction in Japanese patients, while we also revealed its association with ischemic stroke. In addition, another IRAK1 gene polymorphism (Rs1059703) was also related to atherothrombotic cerebral infraction, high CRP, chronic kidney disease, the induction of vaccine-induced immunity and sepsis outcomes but this variant did not differ significantly between our studied groups and controls (data not given). Taking into account that IRAK1 gene affects the activation of transcription factor NF-kB, which is implicated in many immune related genes' expression, we could understand IRAK1 extensive association with many inflammatory conditions beyond patients' origin.

\section{A7.14 IRF5 POLYMORPHISMS IN SYSTEMIC SCLEROSIS}

doi:10.1136/annrheumdis-2013-203221.14

${ }^{1} \mathrm{C}$ Stock, ${ }^{1} \mathrm{H}$ Sato, ${ }^{2} \mathrm{C}$ Fonseca, ${ }^{1} \mathrm{AU}$ Wells, ${ }^{2} \mathrm{CP}$ Denton, ${ }^{2} \mathrm{DJ}$ Abraham, ${ }^{1} \mathrm{G}$ Lindahl, 'EA Renzoni. 'Interstitial Lung Disease Unit, Royal Brompton Hospital, London, UK; ${ }^{2}$ Center for Rheumatology, Royal Free Hospital, London, UK 